\title{
Tratamiento ambulatorio de la trombosis venosa profunda: comparación con tratamiento en hospitalización de pacientes de un registro histórico
}

\section{Outpatient treatment of deep vein thrombosis: comparative study with historical inpatients}

\author{
J. L. Alonso', M. L. Abínzano ${ }^{2}$, M. A. Urbieta ${ }^{1}$, F. J. Anniccherico ${ }^{1}$
}

\section{RESUMEN}

Antecedentes. El tratamiento ambulatorio de la trombosis venosa profunda (TVP) se ha propuesto como un método seguro y coste efectivo, bien completamente ambulatorio o como un patrón mixto de hospitalización corta seguido de tratamiento ambulatorio. El objetivo es comparar la seguridad del tratamiento ambulatorio de la TVP y calcular las estancias medias ahorradas en el hospital.

Material y métodos. Análisis descriptivo de cohortes sobre nuestra experiencia de tratamiento completamente ambulatorio de pacientes con TVP (184 pacientes) durante el periodo 2003 y 2004 -recogiendo las proporciones de hemorragia mayor, complicaciones (definidas como nuevo embolismo pulmonar, re-extensión de la TVP o necesidad de ingreso hospitalario relacionado con TVP) y muerte por todas las causas-, comparadas con una cohorte de 109 pacientes históricos de tratamiento en hospitalización correspondientes al año 2002.

Resultados. De los 184 pacientes tratados ambulatoriamente, presentaron hemorragia mayor 3\% [IC 95\% $1-6,57$, riesgo relativo (RR) 0,38 ], complicaciones $5 \%$ (IC 95\% 2,41-9,37; RR 1,25), de los pacientes y la tasa de mortalidad fue de $4 \%$ (IC $95 \% 1,68-7,99$; RR 0,36 ). Se evitaron 844 estancias hospitalarias. Los 109 pacientes históricos presentaron hemorragia en 8\% (IC 95\% 4-15), complicación $4 \%$ (IC $95 \% 1,18-9,68$ ) y muerte $11 \%$ (IC 95\% 6-18,8).

Conclusiones. El tratamiento completamente ambulatorio de la TVP puede ser tan seguro como el realizado en hospitalización evitándose estancias hospitalarias innecesarias.

Palabras clave. Tratamiento de trombosis venosa profunda. Pacientes ambulatorios. Seguridad del tratamiento anticoagulante.

\begin{abstract}
Background. Outpatient treatment of deep venous thrombosis (DVT) has been proposed as a safe and cost-saving process, either as a mixed pattern: as an inpatient for 1 to 3 days followed by outpatient treatment; or rarely as completely outpatient.
\end{abstract}

Patients and methods. We evaluated two cohorts of consecutive patients diagnosed with DVT. Patients who received entirely outpatient treatment in the years 2003 and 2004, compared with historical patients treated as inpatients during the year 2002. Our aim was to evaluate safety and the days of stay saved because of outpatient treatment of DVT.

Results. A total of 293 patients entered the study (Inpatients, 109; outpatients, 184). Demographic and clinical characteristics of patients were similar. Mean time of anticoagulant therapy and follow up were also both similar in the two groups. Major haemorrhage rate was $8 \%$ (CI 95\% 4-15) in patients treated in hospital and 3\% (CI 95\% 1-6.57) [Relative Risk (RR) 0.38] in patients treated as outpatients. Complications of venous thromboembolic disease occurred in 4\% (CI 95\% 1.18-9.68) of hospitalised patients and 5\% (CI 95\% 2.41-9.37) (RR 1.25) of patients treated as outpatients. The death rate was $11 \%$ (CI $95 \%$ 6-18.8) in hospitalised patients and 4\% (CI 95\% 1.68-7.99) (RR 0.36) in patients treated as outpatients. We observed a reduction of hospitalisation in relation to the index-year of $72.5 \%$ for the year 2003 (CI 95\% -0.08 to -0.04 ) and $79 \%$ for the year 2004 (CI 95\% -0.08 to -0.05$)(\mathrm{p}<0.001)$. Overall, 844 days of unnecessary hospitalisation were saved.

Conclusions. Complete outpatient treatment of DVT shows outcomes at least as safe as inpatient treatment, adding additional reductions in costs for the Health System.

Key words. Therapeutics of Deep Venous Thrombosis. Outpatients. Anticoagulant safety.
1. Servicio de Medicina Interna. Hospital de Navarra. Pamplona.

2. Servicio de Medicina Interna. Hospital Virgen del Camino. Pamplona.

Recepción: 16 de diciembre de 2008

Aceptación provisional: 30 de enero de 2009

Aceptación definitiva: 17 febrero de 2009

\section{Correspondencia:}

José Luis Alonso Martínez

Servicio de Medicina Interna

Hospital de Navarra

Irunlarrea, 3

31008 Pamplona

E-mail: jalonsom@cfnavarra.es 


\section{INTRODUCCIÓN}

Para el tratamiento de la enfermedad trombo-embólica venosa (ETEV), varios estudios han demostrado que las heparinas de bajo peso molecular (HBPM) son tan eficaces como la heparina no fraccionada. Ya que las heparinas de bajo peso molecular no precisan de administración intravenosa y habitualmente no precisan de controles de coagulación, se posibilita el tratamiento de la ETEV de forma ambulatoria ${ }^{1-6}$.

El tratamiento de la trombosis venosa profunda (TVP) consistía clásicamente en el tratamiento en régimen de hospitalización entre 5 y 7 días para la administración de anticoagulantes, pero la introducción en terapéutica de las HBPM ha facilitado el desarrollo del tratamiento ambulatorio de las TVP. Durante los últimos años varios estudios han establecido que el tratamiento ambulatorio de las TVP es probablemente tan eficaz y seguro como el tratamiento en el hospital, siendo también más coste-efectivo ${ }^{7}$. Sin embargo, estos estudios no son homogéneos, algunos de ellos son ensayos clínicos aleatorizados ${ }^{1-6}$, otros son estudios de cohortes $^{8-14}$, varios estudios comparan los resultados clínicos y otros van dirigidos a demostrar el coste efectividad ${ }^{15-22}$. La mayoría de los estudios comparan el tratamiento ambulatorio con HBPM administrada por vía subcutánea con el tratamiento dentro del hospital realizado con heparina no fraccionada, habiendo un número limitado de estudios que comparen la efectividad del tratamiento de la TVP con HBPM dentro y fuera del hospital ${ }^{4}$. Además, de manera importante, en una parte de los estudios publicados, los pacientes pasan entre 1 y 3 días hospitalizados antes de pasar a tratamiento ambulatorio, con lo que la interpretación de los resultados se hace difícil.

En nuestro país son escasos los trabajos publicados sobre tratamiento ambulatorio de la TVP ${ }^{5}$, en general con número de pacientes limitado ${ }^{22-24}$. En este trabajo presentamos nuestros resultados de tratamiento completamente ambulatorio de la TVP y lo comparamos con un registro histórico de tratamiento hospitalario. Los objetivos del estudio fueron evaluar en nuestro medio la seguridad del tratamiento ambulatorio de la TVP y calcular las estancias hospitalarias ahorradas.

\section{MATERIAL Y MÉTODOS}

En nuestro centro, hasta el año 2003, todos los pacientes diagnosticados de TVP eran ingresados en el hospital para tratamiento anticoagulante y reposo absoluto en la cama. En el año 2001 se diseñó un protocolo de actuación en el Servicio de Medicina Interna junto con los servicios de Hematología y de Urgencias, para el tratamiento ambulatorio con HBPM de pacientes diagnosticados de TVP y su seguimiento y fue aplicado durante los años 2003 y 2004. El estudió contó con la aprobación de la dirección del centro y se comunicó la actuación a atención primaria.

El criterio de inclusión para el tratamiento ambulatorio fue el diagnóstico de TVP por ecografía doppler color en el Servicio de Urgencias. A todos los pacientes se les pidió su consentimiento informado para el tratamiento ambulatorio. Se obtuvo así una muestra de 184 pacientes.

Los criterios de exclusión fueron: TVP proximal a la vena femoral, existencia de obesidad mórbida (índice de masa corporal $>40 \mathrm{Kg} / \mathrm{m}^{2}$ ), insuficiencia renal (creatinina plasmática $>2,5 \mathrm{mg} / \mathrm{dl}$ ), datos clínicos de tromboembolismo pulmonar (disnea, dolor torácico, síncope o presíncope o hemoptisis), la falta de soporte social para la administración ambulatoria de los anticoagulantes y la respuesta negativa del paciente al tratamiento ambulatorio.

Los pacientes diagnosticados de TVP iniciaban el tratamiento con HBPM en el Servicio de Urgencias y se les daba una cita para la clínica ambulatoria para los próximos tres días mientras se inyectaban por vía subcutánea enoxaparina una vez al día a la dosis de $1,5 \mathrm{mg} / \mathrm{Kg}$ de peso. En el domicilio la administración de la enoxaparina fue llevada a cabo bien por el grupo de enfermería del centro de salud, bien por auto-administración. Mientras estaban en su domicilio a los pacientes se les permitía estar sentados en un sillón e ir al cuarto de baño.

En la clínica ambulatoria cada paciente fue atendido por uno de los médicos recogiendo historia clínica completa y exploración física, radiografía de tórax, electrocardiograma y análisis rutinarios de sangre y orina. Los pacientes entonces eran asignados a comenzar tratamiento con cumari- 
nas, solapándose con la administración de enoxaparina un mínimo de tres días hasta que el INR de protrombina era $>2$, cesando entonces la administración de enoxaparina. En pacientes discapacitados, con dificultades para asistir a los controles de INR o por elección propia, todo el tratamiento fue realizado con enoxaparina subcutánea.

Se siguió a los pacientes en visitas ambulatorias programadas, y cuando no era posible por dificultades de movilización, el seguimiento se realizó a través del sistema computarizado de eventos clínicos del Servicio Navarro de Salud (que recoge todos los episodios clínicos, visitas a urgencias, cambios terapéuticos y acceso a la historia clínica de atención primaria) y mediante llamadas telefónicas.

Como resultados se recogieron la tasa de hemorragia mayor, definida como la necesidad de transfusión y/o hospitalización, el ingreso relacionado con la ETEV y la muerte por todas las causas. El ingreso relacionado con la ETEV se definió como el causado por embolismo pulmonar, re-extensión de la TVP (definido por trombosis venosa vista por ecografía doppler color en un nuevo territorio venoso) y dolor en la extremidad afectada. La valoración de los pacientes del grupo ambulatorio que precisaron ingreso hospitalario fue hecha por médicos del Servicio deUrgencias.

De un archivo histórico de los pacientes ingresados en el Servicio de Medicina Interna durante el año 2002, se extrajeron para comparación los datos de todos los pacientes cuyo diagnóstico de ingreso fue TVP, que ascendió a un total de 109. Como resultados se recogieron, al igual que para el grupo ambulatorio, la tasa de hemorragia mayor, el reingreso relacionado con la ETEV y el reingreso por dolor, mediante el sistema computarizado de eventos clínicos del Servicio Navarro de Salud y llamadas telefónicas. Todos los pacientes del registro histórico fueron tratados inicialmente con enoxaparina subcutánea a dosis de 1 $\mathrm{mg} / \mathrm{kg}$ de peso cada 12 horas, seguido de cumarinas tras un solapamiento mínimo de tres días.

Para el cálculo de estancias se multiplicó la estancia media de los pacientes del grupo histórico por el número de pacientes ingresados en el año. Ya que todos los pacientes con diagnóstico de TVP fue- ron ingresados en ese año, calculamos el ahorro de estancias en base al año índice restando las estancias ocasionadas por ingresos por TVP en los años 2003 y 2004 para una estancia media similar. No se contabilizó en la disminución de estancias las estancias causadas por hemorragia o complicaciones de la ETEV en ninguno de los dos grupos

\section{Análisis estadístico}

Los resultados se expresan como la media \pm la desviación estándar y como porcentajes. La prueba exacta de Fisher se utilizó para comparar proporciones, la prueba de la $t$ de Student para comparación de medias independientes cuando tenían distribución normal y la prueba de la U de Mann-Whitney cuando tenían distribución no paramétrica. Se calculó el riesgo relativo para ambas cohortes para las variables hemorragia, complicación de la ETEV y muerte. Se empleó la prueba de Log Rank (Mantel Haenszel) para comparar los pacientes libres de eventos durante el tiempo de observación.

Para evaluar la seguridad del tratamiento ambulatorio, comparado con el histórico, se usó un modelo de regresión logística paso a paso, calculando los coeficientes b estandarizados y las odds ratios de las variables que reflejan seguridad (hemorragia, complicación de la ETEV y muerte). Se consideran significativos valores de $\mathrm{p}<0,05$.

\section{RESULTADOS}

Durante los años 2003 y 2004, un total de 184 pacientes fueron tratados ambulatoriamente, 81 (44\%) en el año 2003 y 103 (66\%) en el año 2004. Ninguno de los pacientes que cumplía criterios de inclusión rehusó el tratamiento ambulatorio.

Como grupo comparativo se consideraron todos los pacientes ingresados por TVP durante el año anterior (año 2002) que fueron 109 pacientes. Tres $(1,6 \%)$ de los pacientes incluidos en el grupo de tratamiento ambulatorio, habían sido tratados previamente por ETEV en el grupo histórico. 
En la tabla 1 se muestran los datos demográficos de los pacientes tratados ambulatoriamente y de los pacientes extraídos del archivo histórico con diagnóstico de TVP. La distribución por edad y el sexo fueron similares en ambos grupos así como los antecedentes de ETEV (36 de los pacientes tratados ambulatoriamente habían tenido previamente TVP y 4 tromboembolismo pulmonar; 14 de los pacientes del ar- chivo histórico habían tenido previamente TVP y 6 tromboembolismo pulmonar). La prevalencia de cáncer como factor de riesgo también es comparable tanto en los pacientes tratados ambulatoriamente como en los pacientes extraídos del histórico, sin embargo, la proporción de pacientes con trombosis venosa poplítea fue mayor en los pacientes tratados ambulatoriamente que en los pacientes del histórico.

Tabla 1. Datos demográficos y características de los pacientes tratados por TVP.

\begin{tabular}{|c|c|c|c|c|c|}
\hline & $\begin{array}{c}\text { Pacientes } \\
\text { ambulatorios } \\
\text { (\%) }\end{array}$ & IC $95 \%$ & $\begin{array}{c}\text { Pacientes } \\
\text { históricos } \\
\text { (\%) }\end{array}$ & IC $95 \%$ & $\boldsymbol{p}$ \\
\hline $\mathrm{n}$ & 184 & & 109 & & \\
\hline Edad $_{(\text {años) }}$ & $68 \pm 16$ & & $67 \pm 15$ & & NS \\
\hline Varones & $113(61 \%)$ & $54-68$ & $66(61 \%)$ & $50-69$ & NS \\
\hline Mujeres & $71(39 \%)$ & $32-46$ & $43(39 \%)$ & $30-49$ & NS \\
\hline Antecedentes de ETEV & $40(22 \%)$ & $16-28$ & $20(18 \%)$ & $11,8-27,1$ & NS \\
\hline Días de estancia media & 0 & & $7,75 \pm 3,26$ & & \\
\hline Total de días & 0 & & 844 & & \\
\hline TVP Poplítea & $80(43 \%)$ & $36-51$ & $30(28 \%)$ & $19,6-37$ & $<, 05$ \\
\hline TVP Femoral & $38(21 \%)$ & $15-27$ & $28(26 \%)$ & $18-35$ & NS \\
\hline TVP Femoropoplítea & $60(33 \%)$ & $26-40$ & $43(39 \%)$ & $30-49$ & NS \\
\hline TVP de EE.SS. & $6(3 \%)$ & $1-7$ & $8(7 \%)$ & $3-14$ & NS \\
\hline Cáncer & $23(12,5 \%)$ & $8-18$ & $20(18 \%)$ & $12-27$ & NS \\
\hline $\mathrm{T}^{\mathrm{o}} \mathrm{de}$ anticoagulación ${ }_{\text {(meses) }}$ & $6,72 \pm 4,89$ & & $6,3 \pm 3,56$ & & NS \\
\hline $\mathrm{T}^{\mathrm{o}}$ de seguimiento ${ }_{\text {(meses) }}$ & $12 \pm 7$ & & $12 \pm 6$ & & NS \\
\hline
\end{tabular}

ETEV: Enfermedad tromboembólica venosa. (IC 95\%) Intervalo de confianza al 95\%. NS: valor de p no significativo. Tº: Tiempo. TVP: trombosis venosa profunda. EE.SS: extremidades superiores.

Un total de 844 estancias fueron evitadas por TVP en el periodo de dos años. Observamos para el año 2003 una reducción en la hospitalización por TVP del 72,5\% (IC 95\% $-0,08$ a $-0,04)$ con 30 pacientes tratados en hospitalización frente al histórico de 109, y para el año 2004 una reducción del 79\% (IC $95 \%-0,08$ a $-0,05)$ con 23 pacientes tratados en régimen de hospitalización $(\mathrm{p}<0,001)$.

La tasa de complicaciones se muestra en la tabla 2. Todas las complicaciones ocurrieron en ambos grupos de forma diferida causando ingresos posteriores, resultados ya publicados por nuestro grupo ${ }^{25}$. Episodios de hemorragia mayor ocurrieron en 5 (3\%) de los pacientes tratados ambulatoriamente: 3 presentaron hematoma muscular, 1 hemorragia gastrointestinal y 1 hematuria masiva. En los pacientes del archivo histórico 9 (8\%) tuvieron hemorragia mayor: hematoma muscular 4, hemorragia gastrointestinal 1, epistaxis masiva 1, anemia grave sin otra explicación 2 y hemorragia retrobulbar 1 paciente. Todos los pacientes en ambos grupos precisaron transfusión de concentrado de hematíes. 
Tabla 2. Datos de resultados de pacientes tratados por TVP.

\begin{tabular}{lcccccc}
\hline & \multicolumn{2}{c}{$\begin{array}{c}\text { Pac. ambulatorios } \\
\text { [IC 95\%] }\end{array}$} & $\begin{array}{c}\text { Pac. históricos } \\
{[\text { IC 95\%] }}\end{array}$ & RR & p \\
\hline Hemorragia & $5(3 \%)$ & {$[1-6,57]$} & $9(8 \%)$ & {$[4-15]$} & 0,38 & 0,051 \\
\hline Complicación & $9(5 \%)$ & {$[2,41-9,37]$} & $4(4 \%)$ & {$[1,18-9,68]$} & 1,25 & 0,77 \\
\hline Muerte & $7(4 \%)$ & {$[1,68-7,99]$} & $12(11 \%)$ & {$[6-18,8]$} & 0,36 & 0,029 \\
\hline
\end{tabular}

Pac: Pacientes. RR: Riesgo relativo. TVP: Trombosis Venosa Profunda. IC 95\%: Intervalo de confianza al 95\%.

Complicación: nuevo embolismo pulmonar o re-extensión de TVP, o dolor en la extremidad afecta.

Complicaciones de la ETEV ocurrieron en $9(5 \%)$ pacientes tratados ambulatoriamente: embolismo pulmonar en 3 pacientes, re-extensión de la trombosis en 2 pacientes, dolor precisando analgesia intravenosa en 4 pacientes. Los pacientes del grupo histórico mostraron una tasa de complicaciones de la ETEV del 4\% (cuatro pacientes): embolismo pulmonar 2 pacientes y re-extensión de la trombosis en 2 pacientes.

La tasa de mortalidad fue más alta en la cohorte de pacientes históricos que en el grupo tratado ambulatoriamente.
La tabla 3 muestra los resultados de la regresión logística, con los coeficientes de regresión y los odds ratios de los pacientes tratados ambulatoriamente de la TVP. En el modelo logit no fueron predictivos de eventos la edad, el sexo, la coexistencia de cáncer ni la diferente dosis de enoxaparina. Según el modelo construido el tratamiento ambulatorio fue más seguro para hemorragia y mortalidad total, mientras fue similar para complicaciones de la ETEV.

Tabla 3. Análisis con regresión logística de la seguridad del tratamiento ambulatorio de la TVP.

\begin{tabular}{lcccc}
\hline & b & $\boldsymbol{p}$ & Odds Ratio & IC 95\% \\
\hline Hemorragia & 1,28 & 0,014 & 3,02 & $1,06-9,87$ \\
\hline Complicacion & 0,93 & 0,62 & 0,74 & $0,22-2,46$ \\
\hline Muerte & 1,32 & 0,02 & 3,12 & $1,19-8,20$ \\
\hline
\end{tabular}

IC 95\%: Intervalo de confianza al 95\%. TVP: Trombosis venosa profunda.

Complicación: nuevo embolismo pulmonar o re-extensión de TVP, o dolor en la extremidad afecta.

La figura 1 presenta el análisis Log Rank, mostrando las curvas de pacientes libres de eventos (hemorragia, complicación de la ETEV o muerte) de los pacientes tratados ambulatoriamente y de los pacientes históricos, no mostrando diferencias estadísticamente significativas.

\section{DISCUSIÓN}

Desde la introducción de las HBPM en la terapéutica de la ETEV, se ha producido una derivación del tratamiento de la TVP del hospital al medio ambulatorio. La mayoría de los ensayos disponibles han com- parado el tratamiento de la TVP con heparina no fraccionada en el hospital con HBPM de forma ambulatoria, lo que los convierte en ensayos clínicos abiertos, y en varios de estos ensayos los pacientes del brazo del tratamiento ambulatorio pasan unos pocos días hospitalizados dificultando la interpretación de los resultados. Los estudios publicados realizados con heparinas de bajo peso molecular, pueden ser de difícil interpretación debido a la heterogeneidad de las heparinas utilizadas aun dentro de un mismo estudio; no obstante, se considera que el tratamiento ambulatorio es una alternativa segura al tratamiento convencional en hospitalización. 


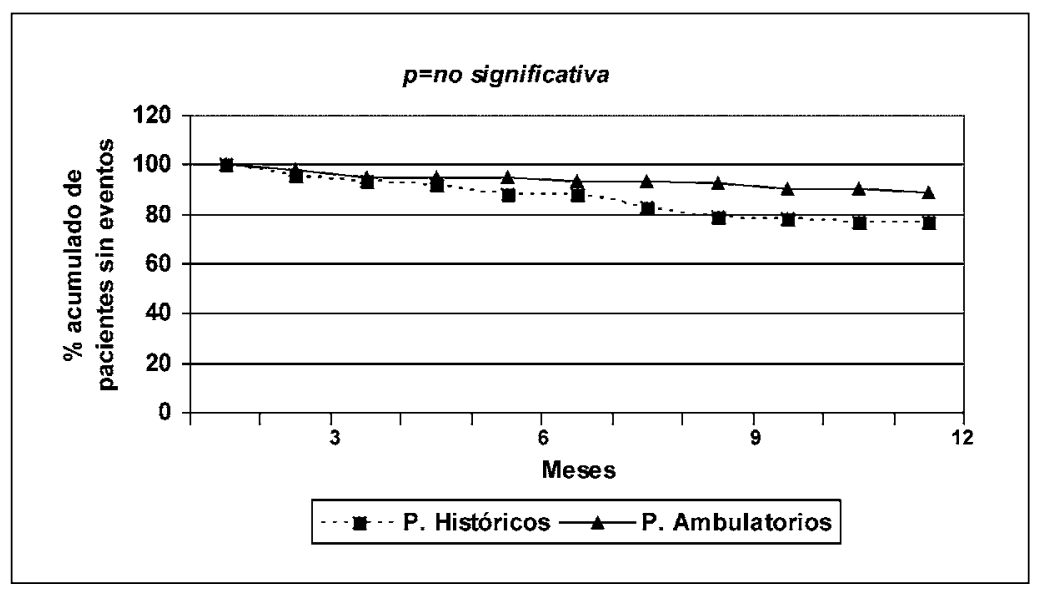

Figura 1. Log Rank de pacientes sin eventos.

Nuestro trabajo es un estudio descriptivo de cohortes con un grupo histórico no seleccionado que incluye todos los pacientes ingresados durante un año natural y con un grupo de tratamiento ambulatorio más seleccionado lo que puede alterar la interpretación de los resultados. No obstante, las características demográficas de los dos grupos, así como la frecuencia de antecedentes de ETEV y de cáncer son similares, haciendo que los dos grupos sean bastante homogéneos favoreciéndose así, en nuestra opinión, la comparabilidad. Los tiempos de anticoagulación y de seguimiento también son similares en ambos grupos, por lo que la observación de complicaciones, hemorragia y muerte en ambos grupos, en nuestra opinión podría ser comparable.

La tasa de complicaciones de la TVP, es decir la re-extensión de la TVP, la ocurrencia de embolismo pulmonar, y el dolor o edema que no se resuelve fue similar en los pacientes tratados ambulatoriamente y en los pacientes históricos, así como la tasa de hemorragia mayor. Sin embargo, la tasa de muerte por todas las causas fue mayor en los pacientes del grupo histórico que en el grupo de pacientes tratados ambulatoriamente. No obstante, los riesgos relativos muestran una magnitud pequeña, pudiendo ser debidos al azar, o a factores de confusión no considerados en el estudio como para poder afirmar la mayor seguridad del tratamiento ambulatorio sobre el tratamiento en hospitalización. En el mismo sentido, la mayor tasa de mortalidad observada en el grupo de pacientes históricos, puede ser debida a la mayor selección en los pacientes de tratamiento ambulatorio, por todo ello, estos datos deben interpretarse con precaución, y en nuestra opinión no significan que el tratamiento ambulatorio cause menos hemorragias mayores o tenga menor mortalidad, ya que con los criterios de inclusión y exclusión, se produce una selección de pacientes para tratamiento ambulatorio que puede explicar estas diferencias. No obstante, la baja tasa de complicaciones de la enfermedad y de su tratamiento parece hacer aconsejable el tratamiento ambulatorio de muchos de los episodios de TVP.

El tratamiento ambulatorio de la TVP ha provocado una disminución de días de estancia hospitalaria por este motivo, reduciéndose las estancias y los ingresos inadecuados, pudiendo ser una práctica clínica coste efectiva resultando en ahorro de costes para este proceso. Aunque en nuestro trabajo no hemos explorado parámetros de calidad de vida, otros estudios han demostrado una mejor calidad de vida y satisfacción del paciente con el tratamiento ambulatorio $^{26}$.

Nuestro estudio, como otros estudios recientes ${ }^{27}$, muestra que el reposo en cama no es necesario en el tratamiento de pacientes con ETEV hemodinámicamente estable, y que la movilización temprana no incrementa el riego de tromboembolismo pulmonar. 
El presente trabajo tiene limitaciones y problemas. Como ya se ha señalado anteriormente, no es un ensayo clínico, es un estudio descriptivo longitudinal y comparativo de cohortes, no está diseñado para observar un efecto directo del tratamiento lo que limita la extrapolación de los resultados a otras poblaciones. Los datos son derivados de una única institución y los pacientes tratados ambulatoriamente tienen una proporción más elevada de trombosis venosa poplítea, que al menos en el terreno teórico, es menos probable que causen tromboembolismo pulmonar. Otro favor limitante es que la dosis de enoxaparina puede no ser directamente comparable ya que en pacientes hospitalizados utilizamos la dosis de $1 \mathrm{mg} / \mathrm{kg}$ de peso dos veces al día, mientras que para los pacientes tratados ambulatoriamente fue diferente, lo que puede explicar las diferentes tasas de hemorragia mayor, sin embargo las dosis de enoxaparina no mostraron ser variables independientes en regresión logística. Además los datos publicados en la literatura ${ }^{28-30}$ sobre la diferente dosificación de enoxaparina, $1,5 \mathrm{mg} / \mathrm{kg}$ una vez al día versus $1 \mathrm{mg} /$ $\mathrm{kg}$ dos veces al día, no han mostrado diferencias en cuanto a eficacia o complicaciones pudiendo ser consideradas similares.

En nuestro trabajo excluimos pacientes con tromboembolismo pulmonar sintomático y pacientes con trombosis venosa iliaca o de cava por lo que los pacientes con trombosis venosa en estas localizaciones probablemente precisen hospitalización para el tratamiento.

No obstante, a pesar de las limitaciones expuestas, parece que el tratamiento ambulatorio de pacientes seleccionados con trombosis venosa profunda es factible en nuestro medio, con un grado de seguridad al menos similar a los pacientes históricos tratados en régimen de hospitalización, más cómoda para los pacientes y disminuyendo los costes del tratamiento de esta enfermedad. Sin embargo, mayor grado de certeza debería obtenerse de un ensayo clínico controlado.

\section{BIBLIOGRAFÍA}

1. Koopman MMV, Prandoni P, Piovella F, Ockelford PA, Brandjes DP, VAn der MEer J et al. Treatment of venous thromboembolism with intrave- nous unfractionated heparin administered in the hospital as compared with subcutaneous low molecular weight heparin administered at home. N Engl J Med 1996; 334: 682-687.

2. Levine M, Gent M, Hirsh J, Leclerc J, Anderson D, Weitz J et al. A comparison of low molecular weight heparin administered primarily at home with unfractionated heparin administered in the hospital for proximal deep vein thrombosis. N Engl J Med 1996; 334: 677-681.

3. O`Brien B, Levine M, William A, Goeree R, Haley $\mathrm{S}$, Blackhouse $\mathrm{G}$ et al. Economic evaluation of outpatient treatment with low molecular weight heparin for proximal vein thrombosis. Arch Intern Med 1999; 159: 2298-2304.

4. Boccalon H, Elias A, Chalé JJ, Cadéne A, GaBRIEL S. Clinical outcome and cost of hospital vs. home treatment of proximal deep vein thrombosis with a low molecular weight heparin. Arch Intern Med 2000; 160: 1769-1773.

5. Grau E, Real E, Pastor E, Viciano V, Aguilo J. Home treatment of deep vein thrombosis: a two years experience of a single institution. Haematologica 1998; 83: 438-441.

6. Belcaro G, Nicolaides AN, Cesarone MR, Laurora $\mathrm{G}$, De Sanctis MT, Incandela L et al. Comparison of low molecular weight heparin administered primarily at home, with unfractionated heparin, administered in hospital and subcutaneous heparin administered at home for deep vein thrombosis. Angiology 1999; 50: 781-787.

7. Vinson DR, BERMAN DA. Outpatient treatment of deep venous thrombosis: a clinical care pathway managed by the emergency department. Am Emerg Med 2001; 37: 251-258.

8. Dunn AS, Schechter C, Gotlin, Vomvolakis D, JAcoBs E, SACKs HS A et al. Outpatient treatment of deep venous thrombosis in diverse innercity patients. Am J Med 2001; 110: 458-462

9. Wells PS, Kovacs MJ, Bormanis J, Forgie MA, Goudie D, Morrow B et al. Expanding elegibility for outpatient treatment of deep venous thrombosis and pulmonary embolism with low molecular weight heparin. A comparison of patient self-injection with homecare injection. Arch Intern Med 1998; 158: 1809-1812.

10. Rose P, Bell D, Green ES, Davenport A, Fegan $\mathrm{C}$, GRECH $\mathrm{H}$ et al. The outcome of ambulatory DVT management using a multidisciplinary approach. Clin Lab Haematol 2001; 23: 301-306.

11. Grau E, Tenias JM, Real E, Medrano J, Ferres R, PASTOR E et al. Home treatment of deep venous thrombosis with low molecular weight heparin: long-term incidence of recurrent venous thromboembolism. Am J Hematol 2001; 67: $10-14$ 
12. Savage HJ, Wells PS, Schulz V, Goudie D, Morrow $\mathrm{B}$, Cruickshank $\mathrm{M}$ et al. Outpatient use of low molecular weight heparin (dalteparin) for the treatment of deep vein thrombosis of the upper extremity. Thromb Haemost 1999; 82: 1008-1010.

13. Schwartz T, Schmidt B, Beyer J, Schroder HE, SCHELLONG SM. Ellegibility for home treatment of deep vein thrombosis: a prospective study in 202 consecutive patients. J Vasc Surg 2001; 34: 1065-1070.

14. Lapius L, Börretzen J, Fahlén M, Thomsen HG, Hasselblom S, Larson L et al. Home treatment of deep vein thrombosis. An outpatient treatment with once-daily injection of low molecular weight heparin (tinzaparina) in 555 patients. Patophysiol Haemost Thromb 2002; 32: 59-66.

15. Segal JB, Bolger DT, Jenckes MW, Krishnan JA, SheIFF MB, Eng J et al. Outpatient therapy with low molecular weight heparin for the treatment of venous thromboembolism: a review of efficacy, safety and costs. Am J Med 2003; 115: 298-308.

16. Spyropoulos AC, Frost FJ, Hurley JS, Roberts M. Costs and clinical outcomes associated with low molecular weight heparin vs. unfractionated heparin for perioperative bridging in patients receiving long-term oral anticoagulation therapy. Chest 2004; 125: 1642-1650.

17. Huse DM, Cummings G, TaYlor DC, Russell MW. Outpatient treatment of venous thromboembolism with low molecular weight heparin: An economic evaluation. Am J Manag Care 2002; 8: S10-16.

18. Lee M, Pao D, Hsu T, Sonderskov A. Cost savings and effectiveness of outpatient treatment with low molecular weight heparin of deep venous thrombosis in a community hospital. Can J Clin Pharmacol 2004; 11: e17-27.

19. Rodger MA, Gagne-Rodger C, Howley HE, CARrIER M, Coyle D, Wells PS. The outpatient treatment of deep venous thrombosis delivers cost savings to patients and their families, compared to inpatient therapy. Thromb Res 2003; 112: 13-18.

20. Spyropoulos AC, Hurley JS, Cierla GN, Lissovoy G. Management of acute proximal deep vein thrombosis: pharmacoeconomic evaluation of outpatient treatment with enoxaparin vs. inpatient treatment with unfractionated heparin. Chest 2002; 122: 108-114.

21. Lindmarker P. Holmstrom M. Use of low molecular weight heparin (dalteparin), once daily for the treatment of deep vein thrombosis. A feasibility and health economic study in an outpatient setting. Swedish Venous Thrombosis Dalteparin Trial Group. J Intern Med 1996; 240: 395-401.

22. Montes J, GonzÁlez L, Amador L, Novo A, Enero M, Rey G et al. Tratamiento domiciliario de la trombosis venosa profunda: Comparación de costes con la hospitalización convencional. An Med Interna (Madrid) 2005; 22: 369-372.

23. Martin Carbonero L, Salgado X, Pedrajas JM et al. Evolución a corto y largo plazo de la trombosis venosa profunda, tratada por una unidad de hospitalziación a domicilio. Rev Clin Esp 2002; 202: 430-434.

24. Suárez Álvarez CG, García Cañete J, Herrero Mendoza MD, Bellver Álvarez TM Arboiro Pinel R. Tratamiento a domicilio de la trombosis venosa profunda con heparinas de bajo peso molecular. Experiencia de un año. An Med Interna (Madrid) 2003; 20: 134-136.

25. Alonso Martínez JL, Abínzano Guillén ML, Urbieta Echezarreta M, Anniccherico SÁnchez FJ, FerNÁNdeZ Ladrón V, García SAnchotena JL. Tratamiento de la trombosis venosa profunda con heparinas de bajo peso molecular. Estudio comparativo con anticoagulación oral. An Med Interna (Madrid) 2008; 25: 4-8.

26. Harrison L, McGinnis J, Crowther M, Ginsberg J, HIRSH J. Assessment of outpatients treatment of deep vein thrombosis with low molecular weight heparin. Arch Intern Med 1998; 258: 2001-2003.

27. Trujillo-Santos J, Perea-Milla E, Jiménez-Puente A, Sánchez-Cantalejo E, Del Toro J, Grau E et al. Bed rest or ambulation in the initial treatment of patients with acute deep vein thrombosis or pulmonary embolism. Chest 2005; 127: 1631-1636.

28. Schobess R, During C, Biduinmaier C, Heinecke A, Merckel N, Nowak-Gotti U. Long-term safety and efficacy data on childhood venous thrombosis treated with a low molecular weight heparin: an open-label pilot study of once-daily versus twice-daily enoxaparin administration. Haematologica 2006; 91 : 1701-1704.

29. Merli G, Spiro TE, Olsson CG, AbildgaArd U, DAvidson BL, Eldor A et al. Subcutaneous enoxaparin once or twice daily compared with intravenous unfractionated heparin for treatment of venous thromboembolic disease. Ann Intern Med 2001; 134: 191-202.

30. Couturaud F, Julian JA, Kearon C. Low molecular weight heparin administered once versus twice daily in patients with venous thromboembolism: a meta-analysis. Thromb Haemost 2001; 86: 980-984. 